\title{
Cost-effectiveness of isoniazid chemoprevention in close contacts
}

\author{
R. Diel*, A. Nienhaus ${ }^{\#}$ and T. Schaberg
}

ABSTRACT: The aim of the present study was to perform a cost-effectiveness analysis in young and middle-aged adults with latent tuberculosis (TB) infection in Germany.

A Markov model simulated the progression of 20 - and 40-yr-old close contacts of active TB cases over $\mathbf{2 0}$ yrs. Health and economic outcomes of isoniazid (INH) chemoprevention versus no intervention were compared. The analysis determined the incremental cost-effectiveness ratio in terms of cost per quality-adjusted life year and the difference between numbers of TB cases and of TB-related deaths.

INH chemoprevention prevented $79 \%$ of expected TB cases in both age groups, and saved $€ 9,482$ and $€ 9,142$ in the lower and higher age groups, respectively, per case prevented. Qualityadjusted life expectancy was slightly extended by 8 days in the lower age group and 7 days in the higher age group, at a cost saving of $€ 417$ and $€ 375$, respectively, per person. Annual savings were $€ 20,862$ and $€ 18,742$ per 1,000 contacts, respectively. The number needed to be treated to prevent one TB case in the lower age group was 23 and 25 in the higher age group. The programme also prevented three TB-related deaths in the younger and two in the older cohort. The results are highly sensitive to treatment-cost assumptions.

In conclusion, isoniazid chemoprevention in Germany is a highly cost-effective approach for reducing the burden of tuberculosis in recently converted young and middle-aged adults.

KEYWORDS: Chemoprevention, cost benefit, cost-effectiveness, Markov simulation, tuberculosis

A fter the decline seen in tuberculosis (TB) incidence from 1991 onwards, it has now re-emerged as a potential threat to public health in Germany, in view of a considerable increase in multi-drug resistance $(2.1 \%$ of all patients suffering from TB disease in 2003). While it is unlikely that the rate of $\mathrm{TB}$, believed to be increasing yet further because of immigration of Eastern European citizens, will be markedly reduced by the establishment of a directly observed treatment strategy alone [1], a monitored isoniazid (INH) chemoprevention programme is being increasingly discussed as a complementary TB control strategy.

Despite the high efficacy of standard INH regimens, as demonstrated in most clinical trials, the cost-effectiveness or cost benefit of such regimens does not seem to be well established. Several pharmaco-economic studies have given inconsistent results and led to contradictory conclusions because of differences in the assumptions on which they were based, primarily concerning the potential of INH to engender serious adverse effects, such as toxic hepatitis [2, 3]. In addition, most studies pertain to USA patients and use cost data and life tables from the USA. Since the costs of preventive regimens and TB case treatment differ substantially throughout the world, their results cannot be applied to patients in Germany [4].

In Germany, INH chemoprevention (300 mg over 9 months) has now been clearly recommended for latent TB infection (LTBI) [5], carefully limiting the range of application to contacts $<50$ yrs of age in order to minimise possible hepatotoxic side-effects. Despite this fact, in most public health bureaus responsible for the observation of close contacts of newly reported cases of $\mathrm{TB}$, recent converters are seldom offered $\mathrm{INH}$, which is, therefore, not routinely implemented in collaboration with the pulmologists who followup these patients. This may be due to fear of unnecessary costs and/or to an imprecise conception of the benefits that can be achieved.

To accelerate decision-making in expanding INH prevention, this study sought to determine its cost-effectiveness within the framework of the German healthcare system. A projection of the expected clinical and economic outcomes of applying chemoprevention to close contacts with LTBI was made. In addition, the costeffectiveness of INH chemoprevention adapted to the recent German guideline was estimated.
AFFILIATIONS

${ }^{*}$ School of Public Health, University of Düsseldorf,

\# Institution for Statutory Accident Insurance and Prevention in the Health and Welfare Services, Hamburg, and "Lungenklinik Unterstedt, Diakoniekrankenhaus Rotenburg (Wümme), Germany.

CORRESPONDENCE

R. Diel

School of Public Health c/o Institute for Medical Sociology Heinrich Heine University

Post box 101007

D-40001 Düsseldorf

Germany

Fax: 492118119769

E-mail:

Roland.Diel@uni-duesseldorf.de

Received:

April 202005

Accepted after revision:

May 272005 


\section{METHODS}

\section{Decision analysis model}

To perform the cost-effectiveness analysis, a Markov model was developed to trace the health outcomes for two hypothetical cohorts of young (20-yr-old) and middle-aged (40-yr-old) adult close contacts of culturally confirmed TB patients. The cohorts were screened by the local public health staff according to $\S 26 \mathrm{ff}$. of the German Contagion Protection Law (Infektionsschutzgesetz (IfSG)), performing Mantoux test with a positive result. Once active TB had been excluded, these patients were considered to be cases of LTBI and were compared prospectively in two sub-groups: 1) those who completed a 9-month course of INH; and 2) those who did not receive INH.

In a Markov model, the natural history of an infection or disease is represented by a set of predefined health states in which a member of the cohort may be at any given time. These states are mutually exclusive (since a patient cannot be in more than one state at any time) and collectively exhaustive. Individuals infected with Mycobacterium tuberculosis can remain clinically healthy while in the state "latent $\mathrm{TB}^{\prime}$. From this, they can move either to the state "death" (with a transition probability equal to the all-cause mortality excluding the disease in question), which it is impossible to leave and from which it is impossible to develop "active $\mathrm{TB}^{\text {" by }}$ reactivation. In the latter state, the patient not only experiences the symptoms of disease, but also has an increased risk of death as a result of the disease over and above the all-cause mortality risk. It is further assumed that TB patients can recover from their disease ("survival") after leaving this state without any relapse during the observation period evaluated in this study. The complete model is depicted in figure 1.

Thus, three transition probabilities (tp) have to be estimated: 1) moving from the latent to the active TB state (tpReact); 2 ) dying when in the latent or active TB state from a condition unrelated to the disease in question (tpDn); and 3) dying when in the active TB state from the disease itself (tpDcm). Time spent by the individuals in a discrete health state is uniformly represented by annual "cycles". For those who complete INH chemoprevention, the annual reactivation probability, tpReact, is reduced owing to the protective INH effect and, thus, has to be multiplied by the complementary probability (1- tpReact). As the INH effect was assumed to last for $20 \mathrm{yrs}$ (see below), the simulation runs for 20 cycles.

A patient's probability of being in one state or another is conditional on the probability of moving from one state to another each year. Therefore, in each subsequent year, the individuals are re-distributed among the different health states. The whole cohort begins at time zero in the LTBI state, at each cycle of the model the appropriate transition probabilities are applied and the distribution of patients in each state is adjusted by normalisation up to the original number of patients. At the end of each cycle the cumulative rewards in terms of outcome measures (quality-adjusted life years (QALY) and cost units per patient) are calculated. As in reality patients will be moving continuously between the different stages and will not just make the transition at the beginning or the end of a cycle, a half-cycle correction was performed.
Probabilities of transitions between states and the effect of INH chemoprevention were determined by Medline literature and the Cochrane Controlled Trials Register review, as they represent the best available data, and they were, where necessary, adapted to the model approach. The corresponding levels of evidence are shown in table 1. To keep the model manageable, the current authors did not consider any reinfections with $M$. tuberculosis as possibly requiring a new course of INH chemoprevention.

\section{Risk of death}

The background likelihood of dying from all causes occurring in the general population is time-dependent. Thus, the probability of transition to disease-unrelated death increases with age in each subsequent year. Data were based on the new German life tables [6] and had, in addition, to be weighted in accordance with the different life expectancies of males and females. At present, mortality due to TB is changing slightly from year to year. In 2002, a total of 7,684 cases fulfilling the case definition were reported in Germany and 513 people died of $\mathrm{TB}$, resulting in a rate of $6.6 \%$ [12]; while in 2003, the corresponding rate was $7.3 \%$ [13]. Therefore, as baseline value, an estimate of $7 \%$ was used.

\section{Effect of INH chemoprevention and compliance}

Most of the available data are from studies assessing a 12month course of INH, with rates of TB reactivation depending primarily upon compliance. There is strong evidence that the benefit of a single-drug INH therapy among close contacts of cases of active TB persists after $\geqslant 19$ yrs $[6,7]$, indicating a reduction in the activation of $\mathrm{TB}$ of up to $90 \%$. Another randomised study of household contacts conducted by the public health service, however, showed only a $68 \%$ protection with a 9-12-month course of INH and based on a compliance (percentage of pills taken) of $\geqslant 80 \%$ [8, 9]. To model "compliance" as an independent entity is difficult, because many possibilities have to be considered, such as noncompliant patients who completely refuse chemoprevention (so that no costs result), but they may also interrupt treatment at any time or take INH irregularly. For this reason, it is inadvisable to choose between the (over-simple and misleading) decision branches "complete chemoprevention" and "incomplete chemoprevention" that could be easily interposed within the option "INH given". It is preferable to incorporate different degrees of compliance with correspondingly different degrees of effect. In addition, the current INH resistance in strains of $M$. tuberculosis, which is nearly $10 \%(7.8 \%$ against $\mathrm{INH}$ on its own plus $2.1 \%$ against INH together with rifampicin [17]) has to be considered. This includes drug resistance among the foreign-born immigrants to Germany.

The present study has accounted for this in the model by decreasing the effect of INH according to the prevalence of INH-resistant TB and, therefore, used $80 \%$ as the baseline protective effect, choosing the corresponding $95 \%$ confidence interval $(\mathrm{CI})$ as the range in the sensitivity analysis. The broad range of $95 \% \mathrm{CI}$ is absolutely necessary since experience shows that healthy people do not tend to adhere to a prolonged drug regimen, and the possibility that poorer compliance will be achieved in some settings has to be taken into account. 


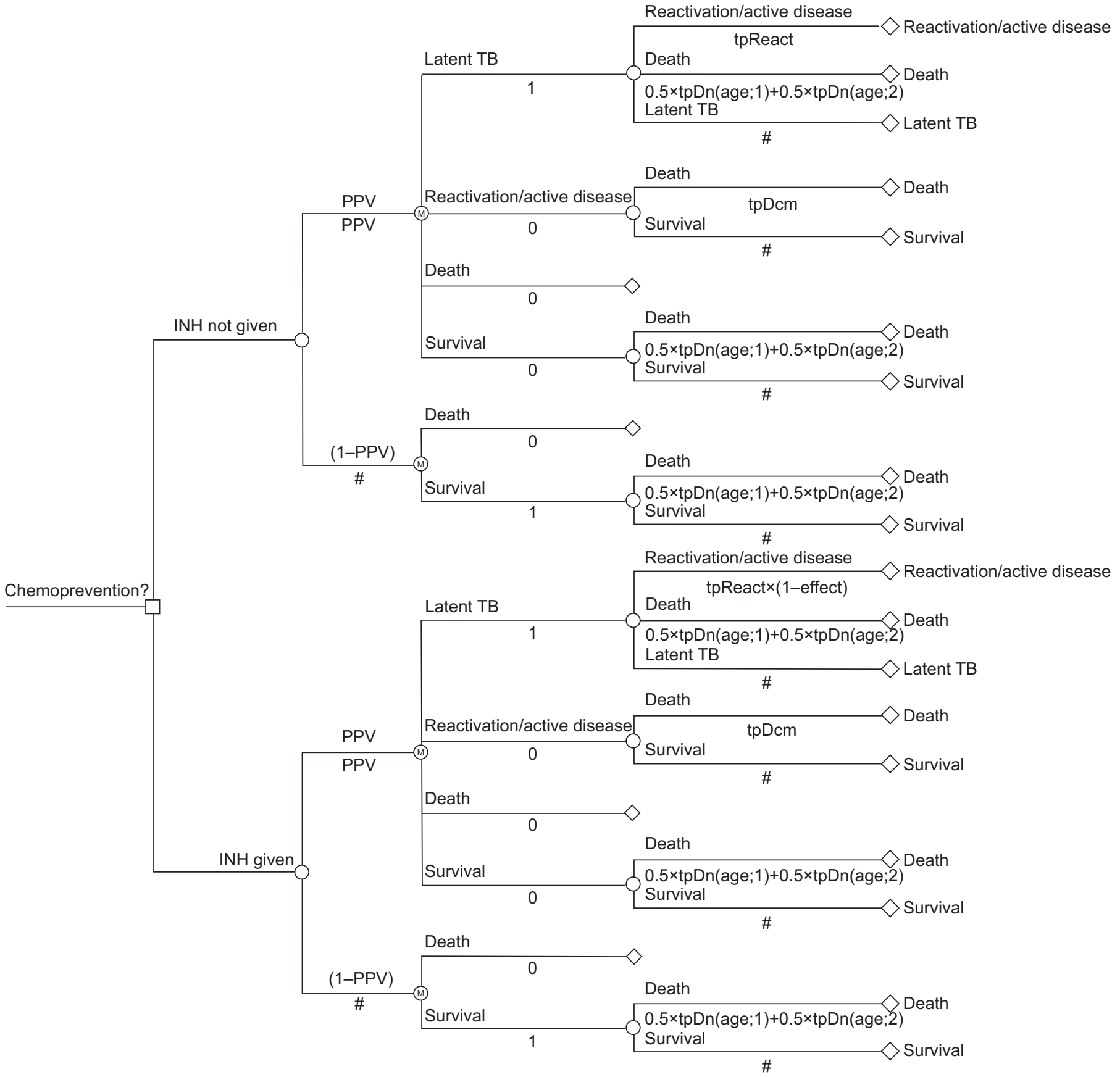

FIGURE 1. Markov model for predicting occurrence of, and deaths from, tuberculosis (TB) due to latent TB. A decision node ( $\square$ ) is the decision after a positive tuberculin skin test to accept isoniazid $(\mathrm{INH})$ or not. A chance node $(\bigcirc)$ represents the possible different health states. A terminal node $(\diamond)$ represents a state from which an individual will jump to the next cycle. Only the state death is an absorbing one and cannot be departed from. PPV: Positive predictive value; tpReact: transition probability for a progression to manifest TB; tpDn: probability of death due to causes other than TB; tpDcm: probability of death due to TB. \#: complementary probability (all probabilities of chance node's branches to sum to 1.0).

\section{TB reactivation rate}

For tuberculin converters, the major risk of development of TB by reactivation is the risk immediately following a recent primary infection. It is estimated that $50 \%$ of LTBI cases who convert do so within the first 2 yrs [18]. A recent meta-analysis [10] showed that the lifetime risk is highly age-dependent, and found a lifetime risk of reactivation TB of 8,10 and $13 \%$ among adults 16-25 yrs of age in three categories of skin-test reactions (induration of at least 5, 10 and $15 \mathrm{~mm}$, respectively). The corresponding risk was 4,5 and $7 \%$ among adults $35-45$ yrs of age. Taking the middle value in each group as a starting value in the present model, and considering the different starting ages of the two cohorts of close contacts, the 20-yr risk of TB reactivation could be translated into a fixed transition 


\begin{tabular}{|c|c|c|c|c|}
\hline Starting age of cohort (age) & 20 and 40 & & & \\
\hline \multicolumn{5}{|l|}{ Prophylaxis variables } \\
\hline $\begin{array}{l}\text { Effect of complete course of INH (annual } \\
\text { reduction in probability of developing TB), } \\
\text { given } \geqslant 80 \% \text { compliance (effect) }\end{array}$ & 0.8 & $0.6-0.9$ & $\mathrm{lb}$ & [6-9] \\
\hline \multicolumn{5}{|l|}{$\begin{array}{l}\text { Annual probability of TB disease with no INH } \\
\text { (tpReact) recent converter }\end{array}$} \\
\hline $16-25$ yrs & 0.0033 & $0.00315-0.00356$ & la & [10] (modified) \\
\hline $36-45$ yrs & 0.0031 & $0.00298-0.00335$ & la & [10] (modified) \\
\hline Prior positive TST/true converter (PPV) & 0.89 & $0.85-0.92^{+}$ & $\mathrm{llb}$ & [11] \\
\hline Nonfatal TB & 0.9 & & Assumption & [15] \\
\hline \multicolumn{5}{|l|}{ Costs $€$} \\
\hline Per course of INH (daily for 9 months) & 201.5 & $100.75-403$ & III & tables 2, 3 \\
\hline Per case of TB & 18850 & 9425-37700 & III & [4] \\
\hline Discount rate \% & 3 & $0-7$ & & [16] \\
\hline
\end{tabular}

INH: Isoniazid; TB: tuberculosis; TST: tuberculin skin test. \#: as outlined by the USA Agency for Health Care Policy and Research (AHCPR) 1992; ": Estimates based on healthcare reimbursement; ${ }^{+}$: $95 \%$ confidence interval.

probability, as follows. 1) Twenty-yr-old contacts. Five per cent risk of disease within 2 yrs after infection, and another $5 \%$ during the remainder of their lifetime. Given a remaining life expectancy of $57 \mathrm{yrs}$ (averaged between males and females), a $\lambda$ value of 0.00090 results [1-exp $(-57 \lambda)$. The risk of reactivation within the following 18 yrs is [1-exp $(-18 \times$ $0.00090)]=1.62 \%$. Thus, the total $20-y r$ risk is $6.62 \%$ (range 6.30-7.12). Divided by 20 cycles, the transition probability is 0.0033 (range $0.00315-0.00356$ ). 2) Forty-yr-old contacts. A $2.5 \%$ risk of disease after infection, and a remaining life expectancy of $38 \mathrm{yrs}$ and a $1.2 \%$ risk in the following $18 \mathrm{yrs}$ of observation, summing up to $6.20 \%$ (range 5.95-6.70). Divided by 20 cycles the transition probability is 0.0031 (range 0.00298 $0.00335)$.

\section{Positive predictive value of contact tracing}

A crucial point in cost-effectiveness analysis is how many individuals offered INH treatment indeed have LTBI, i.e. how many contacts with a positive tuberculin skin test (TST) result are truly infected and may consequently profit from chemoprevention. The positive predictive value (PPV) is estimated by the following formula:

$$
\begin{gathered}
\text { Sensitivity } \times \text { prevalence } /(\text { sensitivity } \times \text { prevalence }+ \\
(1-\text { specificity }) \times(1-\text { prevalence }))
\end{gathered}
$$

Thus, giving a 95\% specificity and a 95\% sensitivity for the Mantoux test, the PPV is $89 \%$ if there is a prevalence of $\mathrm{TB}$ infection of $\geqslant 30 \%$ in the population to be tested. This corresponds to the probability of a TB infection in close contacts of culturally confirmed TB patients [11], and is chosen as baseline value. As there is no generally accepted definition of contact closeness, and because actual prevalence data for contacts in German settings confirmed by molecularepidemiological investigations are lacking, the definition and prevalence data of BEHR et al. [11] were used. Following this, people who spent an estimated total of $\geqslant 40 \mathrm{~h}$ with the index cases in the 3 months before diagnosis or during the infection period were assigned (besides household contacts) to the category of "close contacts". In order to avoid possible overestimation due to uncertainty about the prevalence of infections, the basal PPV was subjected to sensitivity analysis by reducing the assumed base-case prevalence of infections by $\sim 25 \%$.

\section{Health-related quality of life}

As in other infectious diseases, the quality of life in some health states following TB infection varies. Therefore, by convention, quality-adjusted life expectancy (QALE) is estimated by multiplication of the length of life in a discrete health state and the utility of the state which lies between 0 (dead) and 1 (perfect health) on a scale corresponding to (1-disutility). In the absence of any specific tools for estimating QALYs lost due to $\mathrm{TB}$, the literature review is restricted to clinical judgements and clinically based assumptions. Above all, disutility is strongly dependent on the different phases of the disease and has to be weighted on the basis of previous estimates of disutility (1 month spent in hospital with non-fatal TB $[19,20])$ of 0.5 and of 0.1 of the time sick at home or as an outpatient. It is conventional to assess a QALY reduction over $1 \mathrm{yr}$, i.e. one cycle in the model of 0.1 [15]. Accordingly, the QALY was diminished due to TB disease during one cycle in base-case analysis by $10 \%$. 
A 9-month course of INH prevention was not attributed a disutility, because the risk of toxic hepatitis as a side-effect is very small, $\sim 0.15 \%$ of those completing treatment, and is, furthermore, age related [21]. Thus, this side-effect would mostly occur in those aged $>50$ yrs, for whom the German guideline does not recommend INH treatment. Its effect upon quality of life is, therefore, negligible.

\section{Costs and cost-effectiveness}

All costs were reported in 2004 in Euros. The actual cost of TB diagnosis and treatment was recently analysed in a national cost-of-illness study from the German social perspective, measuring both inpatient and outpatient costs (mean combined cost per case $€ 18,850$ ) spent by the health insurance organisation (in Germany, the public Krankenkassen (GKV)), and the indirect costs arising from loss of productivity (mean indirect costs per case $€ 2,461$ ). This analysis is published in detail elsewhere [4]. Costs of measures by the public health service according to $\$ 16$ paragraph 1 (contact tracing) of the IfSG in their function as government institutions (e.g. Mantoux testing and radiographical examinations during the first 2 yrs of observation to the recommendations of the Deutsches Zentralkomitee (German Central Committee for the Control of TB) [22]) are not included because of a lack of valid estimates in this setting, and are reserved for more extensive calculations.

The cost of INH prevention, including professional fees, laboratory tests and medication costs (table 2), are estimated to be $€ 201.50$ per model person. Close contacts undergo chest radiography before beginning prevention and as part of normal contact tracing usually every 3 months either at or on behalf of their local public health centre. As previously described, this cost was not included in the cost of prophylaxis.

TABLE 3 Krankenkassen costs for isoniazid
chemoprevention: medication

Contact persons receiving daily drug regimens need to visit a physician every month for examination, including hepatic, renal and serological monitoring. The presence or absence of a HIV infection, which would require a prolonged (12-month) regimen of INH chemoprevention, has to be determined before the start of INH treatment. INH drug costs were based on the cheapest available drug with the corresponding active substance according to the 2004 issue of the Rote Liste. The package size was adjusted for the number of treatment days (table 3 ).

By convention, different clinical interventions (i.e. in the present model, the strategy of INH chemoprevention versus no prevention), are compared in terms of their incremental cost-effectiveness ratio (ICER), defined as (CA-CB)/(EA-EB), where $C A-C B$ is the difference between the costs of interventions $\mathrm{A}$ and $\mathrm{B}$, and $\mathrm{EA}-\mathrm{EB}$ is the difference between the effectiveness of these interventions. Effectiveness is measured in terms of the number of cases of the disease avoided and QALYs, to yield the net Euro cost required to increase by one of these additional nonmonetary outcome units, compared with the next less costly strategy. Negative numbers, thus, identify savings while positive numbers indicate additional

TABLE 2 Krankenkassen (GKV) costs for isoniazid chemoprevention: medical and technical costs

\begin{tabular}{|c|c|c|c|c|c|}
\hline Medical services & Points n & $\begin{array}{l}\text { Point value } \\
\text { cents }^{\#}\end{array}$ & $\begin{array}{l}\text { Individual } \\
\text { payment } €\end{array}$ & $\begin{array}{l}\text { Frequency of } \\
\text { procedure }\end{array}$ & Payment $€$ \\
\hline \multicolumn{6}{|l|}{ First visit to doctor } \\
\hline Ordination fee per quarter (EBM 1) & 265 & 4.024 & 10.66 & 3 & 31.98 \\
\hline Intensive consultation (EBM 17) & 300 & 4.024 & 12.07 & 3 & 36.21 \\
\hline Whole body status, once per case (EBM 60) & 320 & 4.024 & 12.88 & 3 & 12.88 \\
\hline \multicolumn{6}{|l|}{ Laboratory services } \\
\hline Transport fee per case (EBM 7103) per quarter & & & 2.60 & 3 & 7.80 \\
\hline Creatinine (Jaffe method) (EBM 3670) & & & 0.25 & 10 & 2.50 \\
\hline Urea (EBM 3669) & & & 0.25 & 10 & 2.50 \\
\hline Blood count (EBM 3843) & & & 1.10 & 10 & 11.00 \\
\hline $\begin{array}{l}\text { HIV serology (immunoassay combination test) } \\
\text { EBM } 4543\end{array}$ & & & 4.60 & 1 & 4.60 \\
\hline GOT (EBM 3681) & & & 0.25 & 10 & 2.50 \\
\hline GPT (EBM 3682) & & & 0.25 & 10 & 2.50 \\
\hline
\end{tabular}

EBM: Einheitliche Bewertungsmaßstab (unified GKV cost table used in Germany); GT: glutamyl transpeptidase; GOT: glutamic-oxaloacetic transaminase; GPT: glutamicpyruvic transaminase. \#: if no fixed price is stated average GKV point value for all costs types and service providers as stated in 2003. 
expenditure per outcome unit. The higher the ratio the less cost-effective the intervention. Most generally accepted medical interventions in the USA healthcare system have incremental cost-effectiveness ratios falling below $\sim € 50,000$ per QALY gained [23], so any ratios below this threshold were considered to be cost-effective.

\section{Discounting}

Discounting reflects the higher value of money spent now as opposed to in the future. Similarly, discounting also weighs quality-of-life deficits experienced now (active TB disease plus treatment) more heavily than those experienced in the future. Therefore, both costs and outcomes were discounted by $3 \%$. As studied by WEINSTEIN et al. [16], the results of a sensitivity analysis done by varying the discount rate from $0-7 \%$ were also investigated.

\section{Sensitivity analysis}

Sensitivity analyses were performed to examine the impact of uncertainty surrounding the basic model assumptions. Uncertainty in both clinical and epidemiological probability estimates and cost parameters were explored in order to assess whether the results in the base-case analysis would be robust to a wide range of possible assumptions. Thus, wherever appropriate, univariate (one-way) sensitivity analyses were conducted using 95\% CI for probability estimates or ranges published in the literature. Costs were halved and doubled to obtain lower and upper limits. Multivariate sensitivity analyses were performed on the likelihood of progression to active disease and on treatment costs for TB.

\section{Statistical analyses}

Decision-analytical calculations were performed with TreeAge Pro Healthcare Module 2005 (TreeAge Software Inc., Williamstown, MA, USA). To calculate CI of the number needed to treat, the Pukka method was used [24].

\section{RESULTS}

\section{Base-case analysis}

The Markov model projected the sustained protection rates into 20-yr risks for each strategy. The estimated discounted and undiscounted costs and outcomes are presented in table 4 . INH chemoprevention clearly dominates the non-INH strategy, i.e. chemoprevention is both less costly and associated with a higher QALE. In both cohorts treated (20- and 40-yr-old close contacts), the INH strategy would initially cost $€ 201,500$ per 1,000 close contacts, more than the no-prevention strategy. Compared with no chemoprevention, however, the 9-month INH regimen slightly increased QALE in 20-yr-old contacts truly infected by $\sim 8$ days, at a cost saving of $€ 417$ per contact. It also extended QALE in 40 -yr-old contacts to $\sim 7$ days at a cost saving of $€ 375$. This would result in annual cost savings of $€ 20,862$ and $€ 18,742$, respectively, per 1,000 close contacts.

Under the baseline criteria, INH chemoprevention strategy would prevent 44 and 41 cases of TB per 1,000 close contacts treated, respectively, corresponding to $\sim 79 \%$ of the expected TB cases. Thus, savings per disease case prevented are $€ 9,482$ and $€ 9,142$, respectively. The programme also prevented three TB-related deaths in the younger cohort and two such deaths among the 40-yr-old cohort, among whom the generally higher mortality largely cancels out the specific risk of mortality due to TB. Therefore, INH chemoprevention reduced the 20-yr absolute risk of dying from TB complications by $\geqslant 10 \%$ (20-yrold cohort) and 3\% (40-yr-old cohort). The number needed to be treated in order to prevent one TB case is 23 (95\% CI $16.7-$ $35.4)$ in the younger cohort and 25 (95\% CI 17.6-39.5) in the middle-aged cohort.

\section{Sensitivity analysis}

There were marked differences in the outcomes of the sensitivity analysis (table 4); however, there were no changes in the relative ranking of the two strategies for either costs or outcomes. The conclusions of the base-case analysis were not altered by using the lower or upper CI or range limits for probabilities or costs.

Whereas a doubling in costs of INH chemoprevention will reduce the incremental cost saving of about one-half (-48.3 and $-53.8 \%$ in the 40 -yr-old cohort), a halving in disease costs leads to a reduction in the cost saving by about three-quarters (-74.1 and $-76.9 \%$ ). This may be explained by the fact that a lowering of the high disease costs exceeds by far the increase in INH costs. Thus, the present model results appear to be most sensitive to treatment cost assumptions.

The increase in the effect of chemoprevention from $80-90 \%$, in this case representing both good compliance and absence of INH resistance, results (in both cohorts) in a relative increase of cost savings of $\sim 16 \%$ and six $(12 \%)$ more TB cases averted, but may gain just one more day of QALE. Even a lowering of the effect to $60 \%$ would save $€ 239$ and $€ 229$, respectively per contact, and would avert 33 and 31 TB cases and two TBrelated deaths.

An increase in the discount rate to $7 \%$ would lead to a worsening in the ICER of $\sim 41 \%$. However, the average cost savings per TB case averted as a result of chemoprevention per 1,000 close contacts are still $\sim € 6,118$ and $€ 5,784$. Accordingly, a decrease in the discount rate to $0 \%$ in the sensitivity analysis leads to further cost savings per patient of approximately onethird $(32.8 \%)$.

The threshold value above which the use of INH would be less effective than no chemoprevention would be an increase of $€ 619$ (20-yr-old cohorts) and €577 (40-yr-old cohorts) for the cost of chemoprevention per patient, i.e. approximately three times the base value, and a reduction of treatment cost from $€ 18,850$ to $€ 6,139$ and $€ 6,588$, respectively. Accordingly, threshold probabilities, below which INH would be less effective than no strategy, are a PPV of 0.29 (0.31), a protective effect of $0.265(0.285)$ and a probability of reactivation to active disease of 0.00105 (0.00106).

Even in a worst-case analysis (effect 0.6, INH cost doubled, TB treatment cost halved, probability of reactivation and PPV both at the lower 95\% CI) the resulting ICER of $€ 26,088$ and $€$ 22,692 per QALY fails by far to reach the limit of $\$ 50,000$ described previously as an upper cut-off limit for a costeffective treatment option.

\section{DISCUSSION}

Using a Markov simulation, the natural history of a large cohort with latent TB was predicted. This cohort served as a control for a similar-sized cohort that underwent INH chemoprevention. In 
TABLE 4 Health and economic outcomes

\section{Cases of TB predicted}

Difference (future cases avoided) \%

Effect 0.9

Effect 0.8 (base-case)

PPV 85\% (lower limit of $\mathrm{Cl}$ )

tpReact (lower limit of range)

Effect 0.6

50 (89.3)

44 (78.6)

43

43

$33(58.9)$

$4(14.3)$

$3(10.7)$

$2(7.1)$

Effect 0.6

Life expectancy (QALY) per patient from INH (increment from

INH) rounded days

Effect 0.9

Effect 0.8 (base-case)

Effect 0.6

\section{Costs per patient (increment from INH) $€$}

Effect 0.9

Effect 0.8 (base-case)

Not discounted

Discounted at $7 \%$

Treatment costs halved

Chemoprevention costs doubled

PPV $85 \%$ (lower limit of $\mathrm{Cl}$ )

tpReact lower limit of range

Effect 0.6

Number needed to treat to prevent one TB case

Effect 0.9

Effect 0.8 (base-case)

Effect 0.6

Annual cost per 1000 close contacts (increment from INH) $€$

Effect 0.9

Effect 0.8 (base-case)

Effect 0.6

Incremental cost per case avoided $€$

Effect 0.9

Effect 0.8 (base-case)

Effect 0.6

Worst case scenario

Incremental cost-effectiveness ratio

Threshold analysis"

Decrease of PPV

Reduction of treatment cost $€$

Increase of INH costs $€$

Decrease in effect

Reduction of tpReact

\section{9 (0.025 year) \\ 8 (0.0222 year) \\ 6 (0.0158 year)}

$-496.5$

$-417.2$

$-620.8$

$-244.7$

$-107.9$

$-215.7$

$-390.5$

$-390.0$

$-239.3$

17 (95\% Cl 13.4-22.1)

23 (95\% Cl 16.7-35.4)

31 (95\% Cl 20.0-62.5)

$-24825.50$

$-20862.20$

$-13000.43$

$-9930.2$

$-9482.82$

$-7879.05$

$26,088 € / Q A L Y$

0.29 (prevalence 2.1\%)

6139

618.65

0.265

0.00105
$47(90.4)$

41 (78.8)

40

40

$31(59.6)$

3 (4.2)

2 (2.8)

2 (2.8)

8 (0.0227 year)

7 (0.0201 year)

5 (0.015 year)

$-448.5$

$-374.8$

$-563.1$

$-215.0$

$-86.7$

$-173.3$

$-348.9$

$-353.2$

$-228.5$

21 (95\% Cl 15.9-30.1)

25 (95\% Cl 17.6-39.5)

29 (95\% Cl 19.5-53.7)

$-22427.448$

$-18741.84$

$-11426.87$

$-9543.61$

$-9142.36$

$-6529.64$

22,692 €/QALY

0.31 (prevalence 2.3\%)

6588

576.5

0.285

0.00106

Data are presented as n event per 1,000 close contacts. TB: tuberculosis; PPV: positive predictive value; CI: confidence interval; QALY: quality-adjusted life year; INH: isoniazid; tpReact: moving from the latent to the active TB state. \#: at the same time INH effect 0.6, treatment cost halved, chemoprevention cost doubled, tpReact lower Cl limit, PPV 85\%, discount rate 7\%; ": respective value when "savings" change to "expenditure per QALY" (referring to base-case assumptions).

the simulation, both cohorts were followed for 20 yrs. In controlled therapeutic trials with limited numbers of participants, usually only just enough to satisfy statistical significance, the epidemiological advantages, such as a decline in the incidence and mortality of $\mathrm{TB}$, will only be observed in the next few decades. This powerful modelling tool is also capable of estimating the long-term costs associated with TB and INH chemoprevention as a healthcare intervention. 
Of course, this possible advantage of Markov simulation is counteracted by the weaknesses inherent in a model approach. Even if one tries to take all seemingly relevant parameters into account and provide the best evidence for the source of the parameter estimates drawn from the published literature, such an attempt always has limitations in comparison with using actual data from the country concerned. These, however, are lacking because treatment has not yet been implemented. Therefore, the current authors examined the consequences of varying each parameter used in the analysis or multiple parameters simultaneously to determine their results on costeffectiveness ratios.

Regardless of the assumption, especially considering INH resistance, the model clearly predicts that the implementation of INH chemoprevention in Germany will be more costeffective and less expensive than the current standard approach, i.e. treating of passively diagnosed TB cases together with screening of their contacts, or may be at least be described as cost-effective by convention. This affects young as well as middle-aged contacts with LTBI and is more important, as the (necessarily limited) PPV of the TST screening and the likelihood of uninfected individuals being followed-up as a result of false-positive results was taken into consideration. This aspect is omitted in most of the previously published papers on the cost-effectiveness of INH chemoprevention [3, 20, 25-27].

For the general population in Germany, the present model may underestimate the benefits, as it does not include the number of secondary and tertiary infections averted. Adding these to the decision tree would certainly increase the cost saving obtained from INH, but it would also make this model even more complex. Another important limitation is the fact that the model did not take into account re-infection during the proposed time of protection, especially in highrisk groups where outbreaks occur [28]. However, estimating the probability of re-infection is beyond the scope of the present study, but it could be a topic for future modelling research.

Although the study reveals conspicuous differences in the development of active disease and death following INH chemoprevention and no preventive strategy, thus, providing an economic justification for using INH in Germany, the following issue should be considered when applying the results. A frequently voiced concern is that adding INH to a poorly functioning public health TB control programme would not attain the goal aspired to. Essential for compliance, and therefore critical for the strategy's success, is the willingness of patients with LTBI to complete a 9-month course of treatment. This is a crucial point concerning the validity of the present calculations, especially as recent data in Germany concerning adherence are only available for the treatment of manifesting TB, e.g. $89 \%$ in a metropolis [29]. Adherence to other common preventive treatments, such as prophylactic treatment against hypertension, can only be estimated.

Improving the cohort's adherence to recommended INH chemoprevention is of course the most important mechanism for increasing cost-effectiveness. The local public health staff must serve as an interface between the person with LTBI and the pulmologist to whom this person is referred, especially when it is considered that people in high-risk groups, such as homeless people or drug-abusers, are often not accustomed to participating in insurance-related healthcare programmes. If the supervision of INH clients, beyond initial Mantoux testing and conducting chest radiographs at fixed time intervals, is to become a component of the public health service, this will require additional expenses for staff and cannot, in the short term, be implemented only by an allocation from current resources within the public health bureaus.

In conclusion, in the light of the results of this work, isoniazid chemoprevention appears to offer a worthwhile opportunity to secure better tuberculosis control in Germany. As an added tool, alongside active screening and prompt treatment of tuberculosis disease, it can enable large social cost savings and the prevention of future tuberculosis cases.

\section{REFERENCES}

1 Horsburgh CR. The global problem of multidrug-resistant tuberculosis. The genie is out of the bottle. JAMA 2000; 283: 2575-2576.

2 Fitzgerald JM, Gafni A. A cost-effectiveness analysis of the routine use of isoniazid prohylaxis in patients with a positive Mantoux skin test. Am Rev Respir Dis 1990; 142: 848-853.

3 Salpeter SR, Sanders GD, Salpeter EE, Owens DK. Monitored isoniazid prophylaxis for low-risk tuberculin reactors older than 35 years of age: a risk benefit and cost-effectiveness analysis. Ann Intern Med 1997; 127: 1051-1061.

4 Diel R, Rappenhöner B, Schaberg T. The cost structure of lung tuberculosis in Germany. Eur J Health Econ 2004; 5: 278-284.

5 Schaberg T, Hauer B, Loddenkemper R, et al. Latent tuberculosis infection: recommendations for preventive therapy in adults in Germany. Pneumologie 2004; 58: 92-102.

6 Comstock GW, Baum C, Snider PC Jr. Isoniazid prophylaxis among Alaskan Eskimos: a final report of the Bethel isoniazid studies. Am Rev Respir Dis 1979; 119: 827-830.

7 Smieja MJ, Marchetti CA, Cook DJ, Smaill FM. Isoniazid for preventing tuberculosis in non-HIV infected persons. Cochrane Database Syst Rev 1999; 1: CD001363.

8 Snider DE Jr. Decision analysis for isoniazid preventive chemotherapy: take it or leave it? Am Rev Respir Dis 1988; 137: 2-4.

9 Ferebee SH, Mount FW. Tuberculosis morbidity in a controlled trial of the prophylactiv use of isoniazid among household contacts. Am Rev Respir Dis 1962; 85: 490-521.

10 Horsburgh CR Jr. Priorities for the treatment of latent tuberculosis infection in the United States. $N$ Engl J Med 2004; 350: 2060-2079.

11 Behr A, Hopewell PC, Paz EA, Kamamura LM, Schecter GF, Small PM. Predictive value of contact investigation for identifying recent transmission of Mycobacterium tuberculosis. Am J Respir Crit Care Med 1998; 158: 465-469.

12 Robert Koch Institut. Bericht zur Epidemiologie der Tuberkulose in Deutschland für 2003. [Report on the 
epidemiology of tuberculosis in Germany 2003]. Robert Koch Institute, Berlin 2004.

13 Deutsches Zentralkomitee zur Bekämpfung der Tuberkulose. 28. Informationsbericht. [German Central Committee for control on tuberculosis. 28th information report on tuberculosis]. Berlin 2004.

14 Statistisches Bundesamt. [Federal Statistical Office]. Wiesbaden 2004. Mortality table 2001/2003. www. destatis.de /download/d/bevoe/sterbet03.xls

15 The CEA registry. Standardizing the methods and practices of cost-effectiveness analysis. Table of preference weights for 1998-2991. Harvard Center for risk analysis. www.hcra. harvard.edu/cearegistry/data/phaseIIprefernceweights. pdf

16 Weinstein M, Siegel J, Gold M, Kamlet M, Russell L. Recommendations of the panel on cost-effectiveness in health and medicine. JAMA 1996; 276: 1253-1258.

17 Robert Koch Institut. Tuberkulose in Deutschland 2003. [Tuberculosis in Germany 2003]. Epidemiologisches Bulletin 2004; 44: pp 375-377.

18 Sutherland I. Recent studies in the epidemiology of tuberculosis, based on the risk of being infected with tubercle bacilli. Ad Tuberc Res 1976; 19: 1-63.

19 Schechter CB, Rose DN, Fahs MC. Tuberculin screening: cost-effectiveness analysis of various testing schedules. Am J Prev Med 1990; 6: 167-175.

20 Marchand R, Tousignant $\mathrm{P}$, Chang $\mathrm{H}$. Cost-effectiveness of screening compared to case-finding approaches to tuberculosis in long term care facilities for the elderly. Int $J$ Epidemiol 1999; 28: 563-570.

21 Nolan CM, Goldberg SV, Buskin SE. Hepatotoxicity associated with isoniazid preventive therapy. A 7-year survey from a public health tuberculosis clinic. JAMA 1999 281: 1014-1018.

22 Deutsches Zentralkomitee zur Bekämpfung der Tuberkulose. Richtlinien für die Umgebungsuntersuchungen bei Tuberkulose. [German Central Committee for control of tuberculosis. Guidelines for environmental contact tracing in tuberculosis]. Gesundheitswesen 1996; 58: 657-665.

23 Owens DK. Interpretation of cost-effectiveness analyses. J Gen Intern Med 1998; 13: 716-717.

24 Cook RJ, Sackett DL. The number needed to treat: a clinically useful measure of treatment effect. BMJ 1995; 310: 452-454.

25 Dasgupta K, Schwartzman K, Marchand R, Tennenbaum TN, Brassard P, Menzies D. Comparison of cost-effectiveness of tuberculosis screening of close contacts and foreign-born populations. Am J Crit Care Med 2000; 162: 2079-2086.

26 Macintyre CR, Plant AJ, Hendrie D. The cost-effectiveness of evidence-based guidelines and practice for screening and prevention of tuberculosis. Health Econ 2000; 9: 411-421.

27 Taylor Z. The cost-effectiveness of screening for latent tuberculosis infection. Int J Tuberc Lung Dis 2000; 4: Suppl. 2, S127-S133.

28 Diel R, Meywald-Walter K, Gottschalk R, Rusch-Gerdes S, Niemann S. Ongoing outbreak of tuberculosis in a lowincidence community: a molecular-epidemiological evaluation. Int J Tuberc Lung Dis 2004; 8: 855-861.

29 Diel R, Niemann S. Outcome of tuberculosis treatment in Hamburg: a survey, 1997-2001. Int J Tuberc Lung Dis 2003; 7: 124-131. 